\title{
Fecal Microbiota Transplantation and Its Usage in Neuropsychiatric Disorders
}

\author{
Alper Evrensel, Mehmet Emin Ceylan \\ Department of Psychiatry, Uskudar University, Istanbul, Turkey
}

Fecal microbiota transplantation has a 1700-year history. This forgotten treatment method has been put into use again during the last 50 years. The interest in microbiota-gut-brain axis and fecal microbiota transplantation is rapidly increasing. New evidence is obtained in the etiopathogenesis of neuropsychiatric disorders. There is a large number of experimental and clinical researches in the field of gut-brain axis. There is limited information on fecal microbiota transplantation. Despite this, initial results are promising. It is commonly used in the treatment of gastrointestinal diseases such as Clostridium difficile infection, Crohn's disease, ulcerative colitis. It is also experimentally used in the treatment of metabolic and autoimmune diseases. There are case reports that it is effective in the treatment of autism, Parkinson's disease, multiple sclerosis, chronic fatigue syndrome and irritable bowel syndrome. Its implementation is easy, and it is a cheap and reliable treatment method. However, the long-term risks are unknown. Additionally, standard application protocols have not yet been established. There are a lot of questions to be answered. A university in Turkey has got official permission this year, and started to apply fecal microbiota transplantation. In this review, neuropsychiatric areas of use of fecal microbiota transplantation have been discussed in the light of the current information.

KEY WORDS: Fecal microbiota transplantation; Psychiatry; Neurology; Immune system; Gut-brain axis.

\section{INTRODUCTION}

Microbiota-gut-brain axis and fecal microbiota transplantation (FMT) has been the focus of attention of the scientific community in recent years. More than $90 \%$ of microbiota-themed articles have been published in recent years. ${ }^{1)}$ Gut-brain axis and the role of intestinal microbiota in neuropsychiatric disorders are presented in a previous review. ${ }^{2,3)}$ FMT is the process of transplanting feces from a healthy donor to the receiver's gut in order to recover the impaired intestinal flora. ${ }^{4}$

The related literature has been searched in PubMed, EMBASE and Google Scholar electronic databases by using the terms such as fecal microbiota transplantation (FMT), fecal transplantation, psychiatry, neurology, schizophrenia, schizoaffective disorder, autism, neuropsychiatry and the gut-brain axis. Available article titles, abstracts and full texts have been investigated in details. There are only a few articles on the use of FMT in psychiatric disorders. The aim of this review is to draw attention

Received: September 21, 2015 / Accepted: December 18, 2015 Address for correspondence: Alper Evrensel, MD

Uskudar University, Etiler Clinic, Nispetiye Cad. No:19 Etiler, Beşiktas, 34330, Istanbul, Turkey

Tel: +90-2122701292, Fax: $+90-2122701719$

E-mail: alperevrensel@gmail.com to this interesting topic that appears to open up new horizons in the understanding and treatment of neuropsychiatric disorders in the future. This article is the first in the international neuropsychiatry literature.

\section{MAIN SUBJECTS}

\section{Microbiota-Gut-Brain Axis}

In the human gut, $10^{14}$ (100 trillion) microorganisms are living, and it is 10 times more than the total number of human cells. Microorganisms are estimated to contain 150 times more genes than human genome as well. ${ }^{5,6)}$ Advances in modern life such as the use of antibiotics, vaccination, cleaning products with disinfection and high-calorie diet can bring about significant changes in microbiota. ${ }^{7)}$ According to the hygiene hypothesis proposed in 1989, it has been suggested that there is a causal relation between decreased contact with microorganisms, especially in the childhood, due to increased migration to the cities and increased incidence of allergic diseases. ${ }^{8)}$ According to the old friends hypothesis developed by expanding the limits of hygiene hypothesis beyond allergic disorders, human evolution takes place together with friendly (commensal) microorganisms and in interaction with them (interference of microorganism's metabolites and genetic materi-

(c) This is an Open-Access article distributed under the terms of the Creative Commons Attribution Non-Commercial License (http://creativecommons.org/licenses/by-nc/4.0) which permits unrestricted non-commercial use, distribution, and reproduction in any medium, provided the original work is properly cited. 
als into the human systemic circulation, and triggering epigenomic mechanisms) for thousands of years. 9,10 )

It is pointed out that microbiota plays the decisive role on immune function, ${ }^{11,12}$ and interacts with human cells. ${ }^{13)}$ This interaction takes place through toll-like receptor (TLR)-s, one of the pattern recognition receptor (PRR)-s. ${ }^{14)}$ Interaction between the microbiota and the immune system is not limited thereto. Layer of mucus and tight junction proteins in the intestinal epithelium create a physical barrier for bacteria and foreign antigens. ${ }^{15)}$ Micro damage to the intestinal epithelial wall may occur by the changes of the microbiota, and intestinal epithelial permeability may increase. Thus, the microorganism-induced harmful substances can be released into the systemic circulation and can initiate an immune response. ${ }^{16)}$ This condition is called "leaky gut". 17)

Microbiota appears to play an important role in the etiopathogenesis of neuropsychiatry disorders. Probiotics, prebiotics and fecal transplant are among therapeutic applications by regulating microbiota., ${ }^{3,18)}$

\section{History}

The first known application of feces material for treatment has been performed by Ge Hong, a Chinese physician, in the fourth century. ${ }^{19)}$ It has been orally used under the name of "yellow soup" in patient with severe diarrhea. There are no medical records regarding its use through the subsequent centuries. As from the seventeenth century, it has been used in veterinary medicine, orally and rectally. ${ }^{20)}$ In the Second World War warm camel stool has been used by German soldiers for bacterial dysentery treatment. $^{21)}$ In 1958, FMT has been implemented for the first time in modern medicine. A pseudomembranous enterocolitis case with antibiotic-associated severe diarrhea has been treated through fecal enema. ${ }^{22)}$ Interestingly, it could be revealed after twenty years that Clostridium difficile caused antibiotic-associated pseudomembranous enterocolitis. ${ }^{4)}$ The second case series treated with FMT has been published in $1981 .^{23)}$ When literature is reviewed, it is observed that research on this subject is increasing as from that date. Today, a very high percent of studies on FMT consists of Clostridium difficile infection (CDI) oriented gastroenterology literature.

\section{Donor Selection}

Evidence-based criteria for standard application of FMT are not yet defined. The implementation of the Amsterdam protocol is recommended. ${ }^{21)}$ Despite the absence of any evidence, in the past, the feces-donor should be a healthy person living nearby the patient (spouse or close relative), while recently (especially as from 2011), it is recommended to take feces from a stool bank where reliable and healthy donors are used and frequent health screenings are performed. ${ }^{24)}$ Exclusion criteria for donors are the use of antibiotics in the last three months, intravenous drug use, high risk sexual intercourse history, tattoo or piercing, having infectious, neoplastic, metabolic, allergic or autoimmune diseases. ${ }^{21)}$ Feces material shall be at least $150 \mathrm{~g}$ and shall be obtained within 6 hours before application. $^{21)}$

\section{Preparation of Recipient}

The night before the application, the receiver is recommended to take a mild laxative like magnesie calcinee. One hour before FMT, two tablets of loperamide as antidiarrheal shall be given in order to ensure that the transplanted feces stays at least 4 hours long in intestines. ${ }^{25)}$

\section{Application}

Prior to FMT, feces material shall undergo some processes. Feces is diluted with water, saline or milk. Then, feces suspension is obtained with a household hand mixer. It is passed through gauze or coffee filter to free from solid particles. The resulting suspension is taken to 60-ml syringes. ${ }^{18,21)}$ There are different opinions about the type of the preparation. There are different opinions about with what the feces shall be diluted, and whether it shall be mixed with hand or electrical mixer. It is focused on fine details: FMT containing saline has twice the rate of recurrence than FMT containing water; if electrical mixer is used, the number of anaerobic bacteria decreases due to the air mixed in suspension. ${ }^{21)}$ It has been reported that yogurt, buttermilk, and egg can also be used for dilution. ${ }^{25)}$

In which way the fecal suspension shall be given to the patient is one of the important questions. The literature in particular contains information about the CDI treatment, and the correct application way for gastrointestinal diseases in neuropsychiatric disorders is unknown. Feces suspension can be sent to upper gastrointestinal system via endoscope (esophagogastroduodenoscopy), nasojejunal tube or nasogastric tube, and to lower gastrointestinal system via colonoscopy or retention enema. ${ }^{21)}$ In $75 \%$ of more than 400 reported FMT cases, colonoscopy or enema has been used, and endoscopy, nasojejunal tube or nasogastric tube in $25 \%$. $^{26)}$

\section{Possible Effect Mechanism of FMT}

FMT was investigated mostly in CDI. Because of the 
impairment of intestinal bacterial flora distribution (dysbiosis) due to the use of antibiotics, the intestinal mucosa becomes suitable for CDI. FMT repairs impaired flora. ${ }^{27)}$ There isn't any clinical study that investigated the efficacy of FMT in neuropsychiatric disorders. Therefore, the information obtained from animal experiments can give an idea. Many animal experiments have revealed that significant changes have occurred in the immune response and clinical parameters after the regulation of intestinal bacteria distribution. Giving commensal bacteria to germ free (GF) rat orally increased anti-inflammatory featured interleukin (IL)-10 levels. ${ }^{28)}$ Again, giving Lactobacillus $G G$ to laboratory animals caused an increase in plasma IL-10 levels. ${ }^{29)}$ According to the experiments with rats, in case of disruption of bacterial intestinal permeability, bacterial lipopolysaccharides pass through the systemic blood circulation, TLR-s are induced, and it results in increased production of inflammatory cytokines. ${ }^{30)}$ Experimentally created anxiety and depression in GF mice can be reversed by oral administration of Bifidobacterium infantis. $^{31)}$ There is plenty of Bifidobacterium infantis in neonatal intestine and probiotics, and it is defined as "psychobiotic" because of its antidepressant effect. ${ }^{13)}$ Lactobacillus helveticus and Bifidobacterium longum caused decreased anxiety scores in rats. ${ }^{32)}$

In a double-blind, placebo-controlled and randomized parallel group study performed with healthy volunteers, subjects were given Lactobacillus helveticus R0052 and Bifidobacterium longum or plasebo for a month. Decreased stress scores and decreased urinary free cortisol level were found in subjects who received probiotic bacteria. ${ }^{33)}$ When FMT was applied to the mice on a high fat diet and on normal diet, it was found that mice on normal diet had impairment in intestinal permeability, increased bacterial toxins, increased neuro-inflammation and changes in behavior. It was found that transplanted obese type intestinal microbiota caused immunological and behavioral changes before the development of obesity. ${ }^{34)}$

In the light of such experimental studies, it can be proposed that FMT may reveal beneficial results in treatment of neuropsychiatric disorders through immunological mechanisms as a result of transplanting the healthy intestinal microbiota on the unhealthy microbiota.

\section{FMT in Gastrointestinal Disorders}

It is generally accepted that FMT is effective in the treatment of CDI and inflammatory bowel diseases (ulcerative colitis and Crohn's disease). ${ }^{4,26)}$ In a study with the most large sample size, 317 CDI cases has been ob- served, and the recovery rate after FMT has been reported as $92 \%{ }^{35)}$ Its efficacy in treatment of ulcerative colitis has been found to be $63 \%{ }^{36)}$ While there are successfully treated patients with Crohn's disease after FMT, unresponsive cases have also been reported. Success rate is higher in the ulcerative colitis than in the Crohn's disease. ${ }^{21)}$

\section{FMT in Autoimmune Disorders}

There are publications indicating a relationship between intestinal microbiota changes and autoimmune disorders such as idiopathic thrombocytopenic purpura (ITP), arthritis, systemic lupus erythematosus, Sjogren's syndrome and Hashimoto's thyroiditis. ${ }^{4)}$ For example, Hashimoto's thyroiditis can be triggered by Yersinia enterocolitica. ${ }^{37)}$ In an ulcerative colitis case with comorbid ITP, it has been reported that ITP symptoms has disappeared and platelet levels has been normalized after the application of FMT. ${ }^{4)}$ While there is ample evidence for the relation between the immune system and microbiota, the role of intestinal microbiota dysbiosis in autoimmune disorders wouldn't be something surprising. However, there isn't any other case with autoimmune disorder to whom FMT is applied except the case reported by $\mathrm{Xu}$ et al. ${ }^{4)}$

\section{FMT in Metabolic Disorders}

The only double-blind, randomized controlled study on this subject has been carried out by Vrieze et al. ${ }^{38)}$ Half of to 18 male patients with metabolic syndrome were transplanted their own feces (control group), while the other half were transplanted feces from thin donors (experimental group). The experimental group showed significant increase in insulin sensitivity after six weeks from FMT. In a GF mice experiment, first group received high-fat diet while the second group received $50 \mathrm{mg} / \mathrm{kg}$ olanzapine in addition to high-fat diet, and both groups were monitored for 7 weeks. At the end of the study there were no significant differences in weight gain between the two groups. The researchers concluded that the intestinal microbiota need to be in the presence for olanzapine induced weight gain. When the same experiment was carried out with mice without GF (having intestinal microbiota), it was found that olanzapine changed microbiota in an obesogenic way. Determining that olanzapine had the effect of antibiotics on microbiota was perhaps the most interesting finding of the experiment. ${ }^{39)}$ In the light of these findings, it may be suggested that intestinal microbiota dysbiosis may have a role on the formation of metabolic syndrome, diabetes mellitus and obesity, and its restoration 
through FMT may lead to positive results. Butyrate-producing bacteria of the intestine are thought to play a key role in this process. ${ }^{40)}$

\section{FMT in Neuropsychiatric Disorders}

When the literature was search through, information about the application of FMT in psychiatric disorder such as schizophrenia, schizoaffective disorder, mood disorders, depression and anxiety disorders could not be found. However, there is strong evidence that intestinal microbiota dysbiosis may play an important role in the etiopathogenesis of these disorders. ${ }^{2,3)}$ Neuropsychiatric disorders investigated for FMT effectiveness in the literature are presented below.

\section{Irritable bowel syndrome (IBS)}

There are only three case series of IBS where FMT is tested. In the first one, fifty-patients with IBS and inflammatory bowel disease were included to the study. Patients of $36 \%$ had remission and $16 \%$ subsided. ${ }^{4)}$ As the patients of this study were diagnosed not only with IBS but also with inflammatory bowel disease, the efficacy of FMT cannot be evaluated clearly. Second study was performed with 45 IBS cases with the complaint of chronic constipation. Patients of $89 \%$ recovered fully right after the application, and 18 patients' remission lasted for 19 months. ${ }^{41)}$ In another study, 13 IBS cases were monitored for 11 months after FMT. Well-being of $70 \%$ of the cases remained. ${ }^{42)}$ According to the interpretation of these results, FMT can be an effective therapeutic tool for IBS. ${ }^{43)}$

\section{Chronic fatigue syndrome (CFS)}

In a study, fecal bacteria levels of 43 patients with myalgic encephalomyelitis and CFS and 36 healthy controls were measured and compared. It is found that the level of Gram negative Escherichia coli was significantly decreased in the CFS group when compared to the healthy controls $(92.3 \%$ vs. $49 \%)$. ${ }^{44)}$ Response rate of 60 CFS cases after FMT (bacteriotherapy where 13 non-pathogenic bacteria is applied through colonoscopy) was reported to be $70 \%$. After $15-20$ years, 12 of the patients were contacted and information about their condition was obtained. Seven of 12 patients reported full recovery, and 5 reported that they didn't experience CFS between 1.5 and 3 years. ${ }^{45}$

\section{Multiple sclerosis and myoclonic dystonia}

It was reported that neurologic symptoms of 3 multiple sclerosis patients who were applied FMT because of constipation disappeared, and quality of life improved. It was also reported that complaints of diarrhea of a young female patient who was followed-up with the diagnosis of myoclonic dystonia and chronic diarrhea since the age of six disappeared immediately after the application of FMT, and the symptoms of myoclonic dystonia decreased by $90 \%{ }^{4}{ }^{4}$ Unfortunately, there isn't any other reported case. In all reported four cases, positive results are promising.

\section{Autism}

Increased neuroinflammatory markers have been found in mice exhibiting autistic behaviors due to prenatal valproic acid application. ${ }^{46,47)}$ In an experiment performed by Hsiao et al., ${ }^{48)}$ mice exhibiting autistic behaviors were treated with Bacteroides fragilis. Through tight junction protein expression and cytokine production, permeability impairments in mouse intestine have been repaired by Bacteroides fragilis. Also, increased levels of 4-ethylphenylsulfate, a bacterial metabolite, has been found in the serum of autistic mice. This material has led to the emergence of autistic behavior when infused to normal mice. There isn't any clinical research where FMT is applied to autistic patients. However, there are studies on feces samples. Clostridium species has been found to be more in the feces of autistic children than that of healthy children. ${ }^{49,50)}$ Autistic symptoms of two children has been reported to decline after FMT, and of five autistic children after application of Bacteriodes and Clostridium produced in culture. ${ }^{26)}$

\section{Parkinson's disease}

Common complaint of constipation in patients with Parkinson's disease and the start of constipation-complaint approximately 10 years before the start of the motor symptoms has caused the interpretation that the disease begins in the gut. ${ }^{4)}$ The complaint of chronic constipation of a patient with Parkinson's disease has been reported to be treated with antibiotic treatment (vancomycin, metronidazole and colchicine). Neurological symptoms completely disappeared after 10 months of antibiotic treatment. ${ }^{51)}$ Any FMT application applied to a patient with Parkinson's disease has not been reported yet. However, the case reported by Ananthaswamy ${ }^{51)}$ may be a glimmer of hope for the symptomatic treatment of Parkinson's disease through FMT.

\section{Reliability and Side Effects}

FMT is a fairly reliable application. Serious side effects have not been reported. Some patients has complained about diarrhea, and only few of them constipation, gas and 
abdominal pain on the day of FMT application. ${ }^{21)}$ In a series of 317 cases, Gough et al. ${ }^{35)}$ has reported significant side effects only in three patients (upper gastrointestinal hemorrhage, peritonitis and enteritis). Exacerbation of colitis has been reported in a case after application of FMT for the treatment of ulcerative colitis. ${ }^{52)}$ It has been also reported that a case has been dead due to peritonitis developed after transplantation. ${ }^{53)}$ In a long-term follow-up study, no adverse effects have been reported. ${ }^{54)}$ However, strong evidence that reveal whether there is a relation between FMT application and infection, inflammation and gastrointestinal cancer is needed.

In Turkey, official permission of Clinical Research Ethics Committee, and then from the Department of Ministry of Health Tissue, Organ Transplant and Dialysis Services is required. Intestinal microbiota is defined as "forgotten organ" ${ }^{55)}$ It is observed that The Ministry of Health considers microbiota transplantation in the context of organ transplantation. Uygun and collegues received official permissions for FMT and started with the application this year in Department of Gastroenterology, Gulhane Military Medical Academy (GMMA). ${ }^{56)}$

\section{How do Patients Feel?}

Using fecal material is seen negatively by some of the patients. Women, when compared with men, and youngsters, when compared with elderlies (65 years and over), have been reported to be more reluctant. Nasogastric application method is less preferred than colonoscopy or enema. Forty-eight percent of the patients want the feces transplant procedure to be performed in a hospital, $39 \%$ in doctor's office, and $13 \%$ at home. Patients of $33 \%$ were found to be unwilling to pay for FMT. ${ }^{57)}$

\section{Unanswered Questions}

Although FMT has a history of 1700 years, modern medical experience has been gained in recent years. For this reason, there are many questions to be answered. ${ }^{58)}$ In which disorder and in which direction is microbiota balance impaired? Shall the transplant material be obtained from a donor or shall it be produced in the culture? Shall fresh feces be used or shall it be taken from a stool bank? With what shall the stool be diluted? Which dilution material shall be used in different indications? What is the quantity of the stool to be used? Which method shall be used for application? What is the immunologic response emerging/changing after FMT? Does FMT have longterm risks? Many researches have to be performed in order to reply such questions.

\section{Future Directions}

FMT is a cheesy application. ${ }^{18)}$ For this reason its use may be restricted. Also, as donor health screening takes time, a quick transplant may not be provided in case of emergency. A different method has been tried in a study where a fecal transplantation has been applied for CDI treatment. In this study, after taking stool sample from the donor, it has been centrifuged, and 10\% glycerol has been added for protection against freezing. After placing the fecal material in swallowable capsules, it has been frozen at $-80^{\circ} \mathrm{C}$. Capsules have been placed in $-20^{\circ} \mathrm{C} 1-2$ hours prior to the process, and 15 pieces has been applied to the patients orally for two days. Full remission has been achieved in 14 of 20 patients after 3 days, the procedure has been repeated for the rest 6 patients. ${ }^{59)}$ The level of patient satisfaction for oral capsules method is not known. However, oral application of FMT in the form of capsules to regulate intestinal microbiota after antibiotherapy may become a routine procedure in the future. ${ }^{42)}$

\section{CONCLUSION}

FMT is the most effective treatment method in recurrent CDI. It is easy to apply, cheap and reliable. There are case reports showing its effectiveness in treatment of other diseases and disorders associated with intestinal microbiota impairments. However, there isn't any case report in neuropsychiatric disorders. Only one center (GMMA) has been able to get permission from the Ministry of Health this year in Turkey. In neuropsychiatry literature, interest in the microbiota-gut-brain axis topic has increased rapidly in recent years. In the coming period, the rapid determination of FMT applications and strong evidence with case samples demonstrating its effectiveness in neuropsychiatric disorders are needed.

\section{REFERENCES}

1. Khanna S, Tosh PK. A clinician's primer on the role of the microbiome in human health and disease. Mayo Clin Proc 2014;89:107-114

2. Evrensel A, Ceylan ME. Gut-brain axis: the role of gut microbiota in the psychiatric disorders. Curr Approach Psychiatry 2015;7:461-472.

3. Evrensel A, Ceylan ME. The gut-brain axis: The missing link in depression. Clin Psychopharmacol Neurosci 2015; 13:239-244.

4. Xu MQ, Cao HL, Wang WQ, Wang S, Cao XC, Yan F, et al. Fecal microbiota transplantation broadening its application beyond intestinal disorders. World $J$ Gastroenterol 2015;21:102-111.

5. de Vos WM, de Vos EA. Role of the intestinal microbiome in health and disease: from correlation to causation. Nutr Rev 2012;70 Suppl 1:S45-S56. 
6. Lozupone CA, Stombaugh JI, Gordon JI, Jansson JK, Knight R. Diversity, stability and resilience of the human gut microbiota. Nature 2012;489:220-230.

7. Flint HJ. The impact of nutrition on the human microbiome. Nutr Rev 2012;70 Suppl 1:S10-S13.

8. Strachan DP. Hay fever, hygiene, and household size. BMJ 1989;299:1259-1260.

9. Rook GA. 99th Dahlem conference on infection, inflammation and chronic inflammatory disorders: darwinian medicine and the 'hygiene' or 'old friends' hypothesis. Clin Exp Immunol 2010;160:70-79.

10. Rook GA, Lowry CA, Raison CL. Hygiene and other early childhood influences on the subsequent function of the immune system. Brain Res 2015;1617:47-62.

11. Bäckhed F, Manchester JK, Semenkovich CF, Gordon JI. Mechanisms underlying the resistance to diet-induced obesity in germ-free mice. Proc Natl Acad Sci US A 2007; 104:979-984.

12. Round JL, O'Connell RM, Mazmanian SK. Coordination of tolerogenic immune responses by the commensal microbiota. J Autoimmun 2010;34:J220-J225.

13. Dinan TG, Quigley EM. Probiotics in the treatment of depression: science or science fiction? Aust N Z J Psychiatry 2011;45:1023-1025.

14. Lucas K, Maes M. Role of the Toll Like receptor (TLR) radical cycle in chronic inflammation: possible treatments targeting the TLR4 pathway. Mol Neurobiol 2013;48: 190-204.

15. Borre YE, O'Keeffe GW, Clarke G, Stanton C, Dinan TG, Cryan JF. Microbiota and neurodevelopmental windows: implications for brain disorders. Trends Mol Med 2014;20: 509-518.

16. Hornig M. The role of microbes and autoimmunity in the pathogenesis of neuropsychiatric illness. Curr Opin Rheumatol 2013;25:488-795

17. Maes M, Kubera M, Leunis JC, Berk M. Increased IgA and IgM responses against gut commensals in chronic depression: further evidence for increased bacterial translocation or leaky gut. J Affect Disord 2012;141:55-62.

18. Evrensel A, Ceylan ME. The role of fecal microbiota transplantation in psychiatric treatment. Anatolian J Psychiatry 2015; 16:380.

19. Zhang F, Luo W, Shi Y, Fan Z, Ji G. Should we standardize the 1,700-year-old fecal microbiota transplantation? Am J Gastroenterol 2012;107:1755.

20. Borody TJ, Warren EF, Leis SM, Surace R, Ashman O, Siarakas S. Bacteriotherapy using fecal flora: toying with human motions. J Clin Gastroenterol 2004;38:475-483.

21. Smits LP, Bouter KE, de Vos WM, Borody TJ, Nieuwdorp M. Therapeutic potential of fecal microbiota transplantation. Gastroenterology 2013;145:946-953.

22. Eiseman B, Silen W, Bascom GS, Kauvar AJ. Fecal enema as an adjunct in the treatment of pseudomembranous enterocolitis. Surgery 1958:44:854-859.

23. Bowden TA Jr, Mansberger AR Jr, Lykins LE. Pseudomembraneous enterocolitis: mechanism for restoring floral homeostasis. Am Surg 1981;47:178-183.

24. Di Bella S, Drapeau C, García-Almodóvar E, Petrosillo N. Fecal microbiota transplantation: the state of the art. Infect Dis Rep 2013;5:e13.

25. Demirci H, Uygun A. [Fekal transplantasyon nasll ve kime uygulanmall?]. Güncel Gastroenteroloji 2014;18:444-447. Turkish.

26. Aroniadis OC, Brandt LJ. Fecal microbiota transplantation: past, present and future. Curr Opin Gastroenterol 2013;29.
79-84.

27. Bakken JS, Borody T, Brandt LJ, Brill JV, Demarco DC, Franzos MA, et al; Fecal Microbiota Transplantation Workgroup. Treating Clostridium difficile infection with fecal microbiota transplantation. Clin Gastroenterol Hepatol 2011:9:1044-1049.

28. Macpherson AJ, Uhr T. Gut flora--mechanisms of regulation. Eur J Surg Suppl 2002;(587):53-57.

29. Kopp MV, Goldstein M, Dietschek A, Sofke J, Heinzmann A, Urbanek R. Lactobacillus $G G$ has in vitro effects on enhanced interleukin-10 and interferon-gamma release of mononuclear cells but no in vivo effects in supplemented mothers and their neonates. Clin Exp Allergy 2008;38:602-610.

30. Ait-Belgnaoui A, Durand H, Cartier C, Chaumaz G, Eutamene H, Ferrier L, et al. Prevention of gut leakiness by a probiotic treatment leads to attenuated HPA response to an acute psychological stress in rats. Psychoneuroendocrinology 2012;37:1885-1895.

31. Desbonnet L, Garrett L, Clarke G, Kiely B, Cryan JF, Dinan TG. Effects of the probiotic Bifidobacterium infantis in the maternal separation model of depression. Neuroscience 2010;170:1179-1188.

32. Messaoudi M, Lalonde R, Violle N, Javelot H, Desor D, Nejdi A, et al. Assessment of psychotropic-like properties of a probiotic formulation (Lactobacillus helveticus R0052 and Bifidobacterium longum R0175) in rats and human subjects. Br J Nutr 2011;105:755-764.

33. Messaoudi M, Violle N, Bisson JF, Desor D, Javelot H, Rougeot C. Beneficial psychological effects of a probiotic formulation (Lactobacillus helveticus R0052 and Bifidobacterium longum R0175) in healthy human volunteers. Gut Microbes 2011;2:256-261.

34. Bruce-Keller AJ, Salbaum JM, Luo M, Blanchard E 4th, Taylor CM, Welsh DA, et al. Obese-type gut microbiota induce neurobehavioral changes in the absence of obesity. Biol Psychiatry 2015;77:607-615.

35. Gough E, Shaikh H, Manges AR. Systematic review of intestinal microbiota transplantation (fecal bacteriotherapy) for recurrent Clostridium difficile infection. Clin Infect Dis 2011;53:994-1002.

36. Anderson JL, Edney RJ, Whelan K. Systematic review: faecal microbiota transplantation in the management of inflammatory bowel disease. Aliment Pharmacol Ther 2012;36:503-516.

37. Corapçioğlu D, Tonyukuk V, Kiyan M, Yilmaz AE, Emral R, Kamel N, et al. Relationship between thyroid autoimmunity and Yersinia enterocolitica antibodies. Thyroid 2002;12: 613-617.

38. Vrieze A, Van Nood E, Holleman F, Salojärvi J, Kootte RS, Bartelsman JF, et al. Transfer of intestinal microbiota from lean donors increases insulin sensitivity in individuals with metabolic syndrome. Gastroenterology 2012;143:913-916.e7.

39. Morgan AP, Crowley JJ, Nonneman RJ, Quackenbush CR, Miller CN, Ryan AK, et al. The antipsychotic olanzapine interacts with the gut microbiome to cause weight gain in mouse. PLoS One 2014;9:e115225.

40. Udayappan SD, Hartstra AV, Dallinga-Thie GM, Nieuwdorp M. Intestinal microbiota and faecal transplantation as treatment modality for insulin resistance and type 2 diabetes mellitus. Clin Exp Immunol 2014;177:24-29.

41. Borody TJ, Paramsothy S, Agrawal G. Fecal microbiota transplantation: indications, methods, evidence, and future directions. Curr Gastroenterol Rep 2013;15:337.

42. Borody TJ, Brandt LJ, Paramsothy S. Therapeutic faecal microbiota transplantation: current status and future 
developments. Curr Opin Gastroenterol 2014;30:97-105.

43. Pinn DM, Aroniadis OC, Brandt LJ. Is fecal microbiota transplantation (FMT) an effective treatment for patients with functional gastrointestinal disorders (FGID)? Neurogastroenterol Motil 2015;27:19-29.

44. Frémont M, Coomans D, Massart S, De Meirleir K. Highthroughput $16 \mathrm{~S}$ rRNA gene sequencing reveals alterations of intestinal microbiota in myalgic encephalomyelitis/chronic fatigue syndrome patients. Anaerobe 2013;22:50-56.

45. Borody TJ, Nowak A, Finlayson S. The GI microbiome and its role in chronic fatigue syndrome: A summary of bacteriotherapy. J Australas Coll Nutr Env Med 2012;31:3-8.

46. de Theije CG, Wopereis H, Ramadan M, van Eijndthoven $\mathrm{T}$, Lambert J, Knol J, et al. Altered gut microbiota and activity in a murine model of autism spectrum disorders. Brain Behav Immun 2014;37:197-206.

47. de Theije CG, Koelink PJ, Korte-Bouws GA, Lopes da Silva $\mathrm{S}$, Korte SM, Olivier B, et al. Intestinal inflammation in a murine model of autism spectrum disorders. Brain Behav Immun 2014;37:240-247.

48. Hsiao EY, McBride SW, Hsien S, Sharon G, Hyde ER, McCue $\mathrm{T}$, et al. Microbiota modulate behavioral and physiological abnormalities associated with neurodevelopmental disorders. Cell 2013;155:1451-1463.

49. Finegold SM, Molitoris D, Song Y, Liu C, Vaisanen ML, Bolte E, et al. Gastrointestinal microflora studies in late-onset autism. Clin Infect Dis 2002;35(Suppl 1):S6-S16.

50. Song Y, Liu C, Finegold SM. Real-time PCR quantitation of clostridia in feces of autistic children. Appl Environ Microbiol 2004;70:6459-6465.
51. Ananthaswamy A. Faecal transplant eases symptoms of Parkinson's disease. New Sci 2011;209:8-9.

52. De Leon LM, Watson JB, Kelly CR. Transient flare of ulcerative colitis after fecal microbiota transplantation for recurrent Clostridium difficile infection. Clin Gastroenterol Hepatol 2013;11:1036-1038.

53. Aas J, Gessert CE, Bakken JS. Recurrent Clostridium difficile colitis: case series involving 18 patients treated with donor stool administered via a nasogastric tube. Clin Infect Dis 2003;36:580-585.

54. Brandt LJ, Aroniadis OC, Mellow M, Kanatzar A, Kelly C, Park $\mathrm{T}$, et al. Long-term follow-up of colonoscopic fecal microbiota transplant for recurrent Clostridium difficile infection. Am J Gastroenterol 2012;107:1079-1087.

55. O'Hara AM, Shanahan F. The gut flora as a forgotten organ. EMBO Rep 2006;7:688-693.

56. Cetin R, Guven GB, Tuncbilek V, Develi S, Aykutlug O, Korkmaz A. Microorganisms and their interaction with human body. TAF Prev Med Bull 2015;14:273-278.

57. Brandt LJ, Aroniadis OC. An overview of fecal microbiota transplantation: techniques, indications, and outcomes. Gastrointest Endosc 2013;78:240-249.

58. Vandenplas Y, Pierard D, De Greef E. Fecal microbiota transplantation: Just a fancy trend? J Pediatr Gastroenterol Nutr 2015;61:4-7.

59. Youngster I, Russell GH, Pindar C, Ziv-Baran T, Sauk J, Hohmann EL. Oral, capsulized, frozen fecal microbiota transplantation for relapsing Clostridium difficile infection. JAMA 2014;312:1772-1778. 